\title{
Representaciones sociales del noviazgo, en adolescentes escolarizados de estratos bajo, medio y alto, en Bogotá
}

\section{Social representations of having a boy/girlfriend in adolescents still in school from low, middle and high social strata in Bogotá}

\author{
Lilián Sánchez ${ }^{1}$, María E. Gutiérrez², Nelson Herrera ${ }^{3}$, Magnolia Ballesteros², \\ Romina Izzedin ${ }^{4}$ y Ángela Gómez ${ }^{5}$ \\ 1 Universidad Antonio Nariño. Huila- Colombia. liliasanchez@uan.edu.co \\ 2 Universidad Nacional de Colombia, Bogotá. megutierrezd@unal.edu.co,mdballesterosc@unal.edu.co \\ 3 Universidad Antonio Nariño. Bogotá, Colombia. ehm21@ hotmail.com \\ 4 Fundación Universitaria Los Libertadores. Bogotá, Colombia. rizzedinb@libertadores.edu.co \\ 5 Vigilancia en Salud Pública, Instituto Nacional de Salud. Bogotá, Colombia. angela.gomezs@ nuevaeops. \\ com.co
}

Recibido 19 Junio 2010/Enviado para Modificación 30 Noviembre 2010/Aceptado 10 Diciembre 2010

\section{RESUMEN}

Objetivos Abordar el significado del noviazgo mediante la teoría de las representaciones sociales. Determinar las convergencias y divergencias de los participantes en torno a los núcleos figurativos de la representación

Métodos Aproximación cualitativa interpretativa. En tres colegios diferenciados por estrato, se utilizaron observación, red de asociaciones, entrevista en profundidad y talleres grupales. La triangulación intra estrato, intra género y de totalidad de los participantes, permitió verificar los resultados convergentes y divergentes.

Resultados En los tres estratos, el noviazgo hace parte del proyecto de vida centrado en independencia de la familia, éxito laboral y conformación de familia en la vida adulta. El noviazgo no es prioritario y constituye una experiencia de aprendizaje afectivo. La sexualidad coital es concebida como una posibilidad postergable dados los riesgos de embarazo. Las diferencias por estrato, se centraron en los obstáculos o facilidades económicas para realizar las metas. Las diferencias por género se encontraron entre los estratos bajo y alto. Las mujeres de estrato bajo, son más conservadoras, resienten mayor control social y pérdida de independencia. En cambio las del alto, reclaman independencia y critican los modelos tradicionales. Los hombres de estrato bajo, son más tradicionalistas que los de estrato alto y el sufrimiento está ligado a la infidelidad.

Discusión Se destaca la responsabilidad en el diseño de políticas sociales que permitan crear y apoyar los caminos que estos jóvenes transiten hacia el logro de sus metas futuras, en especial a los adolescentes del estrato socioeconómico bajo, quienes enfrentan desventajas económicas, sociales y culturales.

Palabras Clave: Percepción social, adolescente, sexualidad, investigación cualitativa (fuente: DeCS, BIREME). 


\section{ABSTRACT}

Objectives Studying the significance of having a boy/girlfriend by social representations theory. Identifying participants' convergence and difference around a figurative nuclei, focusing on representation by socio-economic strata and gender.

Methods A qualitative interpretative approach was adopted. Three schools from different socio-economic strata were used for observation, network association, indepth interviews and group workshops. Intra-strata, intra-gender and participant triangulation allowed convergent and divergent results to be verified.

Results Having a boy/girlfriend (courtship) in all three strata was considered part of adolescents' life projects aimed at becoming independent from the family, successfully obtaining a job and forming a family in future adult life. Thus, having a boy/girlfriend was not a priority and was seen more as an emotional learning experience. Sexuality was seen as something to be postponed due to the risks of pregnancy. Differences by strata focused on economic obstacles to or chances for achieving goals. Gender differences were found between low and high strata. Girls having low economic status were more conservative, resented greater social control and their loss of independence compared to girls from high socioeconomic strata who demanded their independence and criticised traditional models. Boys having low economic status were more traditional than their upper strata counterparts and the suffering involved in having a girlfriend was linked to infidelity. Suffering in having a boy/girlfriend was linked to such relationship breaking up for upper and middle strata. Masculinity regarding having a boy/girlfriend was affirmed in all strata.

Discussion Emphasis should be placed on designing social policies helping these young people to achieve their future goals, especially teenagers from lower socioeconomic strata who face economic, social and cultural disadvantages.

Key Words: Social perception, adolescent, sexuality, qualitative research (source: $\mathrm{MeSH}, \mathrm{NLM}$.

$\mathrm{E}$ n Colombia, hay preocupación por la formación de conductas sexuales saludables de adolescentes, debido a altos índices de enfermedades de transmisión sexual y a embarazos de adolescentes. La Ley general de educación de 1993 (1), reconoce la necesidad de desarrollar dentro de su propuesta de formación integral, el Proyecto de Educación Sexual. Sin embargo, de acuerdo al boletín del DANE (2), del CENSO realizado en el año 2005, se evidencia que los nacimientos del grupo de 15 a 19 años se han incrementado en un 19,7\%. Siendo las adolescentes de nivel socioeconómico bajo quienes presentan una mayor propensión a quedar embarazadas por iniciar relaciones sexuales más temprano y forman uniones en mayor proporción que las de estrato alto (3-5).

La dimensión afectiva, en el contexto de las relaciones amorosas románticas, es un aspecto a estudiar en la sexualidad adolescente ya que éstas se reconocen 
como experiencias de socialización, con gran significado en el contexto propicio para el inicio de su actividad sexual coital. El noviazgo en adolescentes, presenta un interés creciente en los últimos años en Colombia, después de no haber sido considerado en el estudio de la sexualidad (6-10).

El noviazgo se ha integrado como una dimensión valiosa dentro de la pareja ${ }^{1}$. Además, es considerado como una expresión de la sexualidad, pero en la adolescencia, es visto como un "riesgo", por la vulnerabilidad a un embarazo, ya que la proximidad física se convierte en la principal forma de expresar y recibir afecto (11). No obstante, otra perspectiva en psicología, propone a los noviazgos como oportunidades de crecimiento personal y de aprendizaje sobre uno mismo en relación con los demás (12).

Para los propósitos de este estudio, el "noviazgo" es un concepto que refiere a la experiencia romántica, de vinculación, compromiso y apoyo, de una pareja, en el marco de un contexto social y cultural. Aproximarse al noviazgo implica entonces, conocer los sentidos que los adolescentes otorgan a sus relaciones amorosas y al conocimiento que es compartido sobre el mismo en determinados contextos sociales.

Abordamos el estudio de las representaciones sociales desde una perspectiva cualitativa, interpretativa procesual (13). Este marco conceptual obedece a una elección epistemológica, ontológica y metodológica para construir conocimiento, sustentado en los aportes de Moscovici (14) y Jodelet (15). Las representaciones sociales de noviazgo se analizan en relación con las dinámicas individuales y sociales, en una actividad de construcción y reconstrucción de la realidad, donde lo social se contextualiza en la interacción de los adolescentes. Es así, como se estudian experiencias particulares, no generalizables, que darán cuenta de una realidad construida por el sujeto, que puede facilitar la comprensión de los significados y prácticas de otros adolescentes en contextos similares. El objetivo del presente estudio es reconstruir las representaciones sociales sobre el noviazgo, convergencias y diferencias según estrato social y género.

\footnotetext{
${ }^{1}$ Ramírez J, Bacca A. Representaciones y prácticas en el campo de las relaciones de pareja en Bogotá en el siglo XX: Tránsitos entre la tradición y la modernidad. Tesis de Grado. Programa de Sociología. Facultad de Ciencias Humanas. Universidad Nacional de Colombia; 2003. Se encuentra en la Biblioteca Central.
} 


\section{METODOLOGÍA}

La investigación es multi metodológica, es decir, que la información se obtuvo a través de: observación en la cotidianidad escolar, talleres constructivistas, Red de Asociaciones (16), y entrevistas abiertas (17). La información obtenida con cada instrumento fue triangulada, buscando aspectos convergentes por género y estrato. La triangulación general permitió definir cuál es la representación social del noviazgo para cada uno de los estratos y grupos.

Participantes. Del colegio de estrato bajo participaron 22 adolescentes (9 hombres y 13 mujeres). Del estrato socioeconómico medio, 12 participantes (7 hombres y 5 mujeres) y del colegio de estrato socioeconómico alto, 9 participantes (3 hombres y 6 mujeres).

Se cumplieron las normas éticas consagradas en la ley 1090/2006 (18) que reglamenta la profesión de la psicología. Entre éstas se incluyó el consentimiento informado a los representantes legales de los adolescentes e igualmente se siguieron las normas éticas con las instituciones educativas.

\section{RESULTADOS}

Coinciden los estratos bajo, medio y alto en la representación de los aspectos positivos del noviazgo, en la ambivalencia en la sexualidad dentro del mismo, en las características que más valoran en la pareja y frente al momento más propicio para fundar una familia (Cuadro 1).

Cuadro 1. Representaciones sociales del noviazgo para los tres estratos

\begin{tabular}{ccc}
\hline Mujeres & Hombres & Hombres \\
\hline Relación amorosa consolidada & Experiencia significativa \\
Control social & Ambivalencia actividad sexual & Reafirmación \\
& Proyecto de vida /educación/autonomía/ familia/ \\
hijos/ & masculinidad \\
\hline
\end{tabular}

Para los adolescentes de los estratos socio económico bajo, medio y alto el noviazgo es una experiencia no trascendental. Está inmerso en el proyecto de vida y no ocupa un lugar protagónico a corto y mediano plazo, como la continuidad de los estudios, el trabajo y la búsqueda de estabilidad económica futura.

El proyecto de vida es el referente conceptual desde donde es entendido el noviazgo, como experiencia que se inscribe al proceso de conformación de 
identidad de los adolescentes. La mayoría de aquellos del estrato socio económico bajo aspiran a continuar sus estudios, a ingresar a universidades públicas o instituciones de capacitación técnica como el SENA. Las dificultades económicas les plantean la necesidad de trabajar para poder estudiar. Así el trabajo remunerado cobra un valor fundamental. Por su parte, los adolescentes del estrato socio económico medio desean hacer una carrera, contemplando como primera opción las universidades públicas, ya que las privadas, implican mayores gastos. En la adultez, vislumbran una vida laboral, en actividades cualificadas que garanticen: independencia económica, vivir cómodamente, disfrutar la vida y consolidar su proyecto. Algunos ven el futuro, en otros países, donde esperan estudiar y realizar una nueva vida. Los adolescentes de estrato alto privilegian los estudios profesionales, en universidades privadas reconocidas o fuera del país. Ellos aspiran a ubicarse bien laboralmente para mantener su estilo de vida e independizarse. La actividad laboral se posterga hasta la culminación de sus estudios profesionales. El trabajo para algunos de ellos está garantizado en empresas donde sus padres son propietarios. Otros se plantean la posibilidad de crear sus propias empresas o negocios. Esta perspectiva laboral, en la que se espera que los adolescentes sean generadores de empresa, la promueve el colegio y es una expectativa también de la familia: que sus hijos ocupen cargos de dirección y no sean subalternos (Cuadro 2).

Cuadro 2. Representaciones sociales del noviazgo según estrato social

\begin{tabular}{|c|c|c|c|c|}
\hline Estrato bajo & \multicolumn{2}{|c|}{ Estrato medio } & \multicolumn{2}{|c|}{ Estrato alto } \\
\hline Hombres $\quad$ Mujeres & Hombres & Mujeres & Hombres & Mujeres \\
\hline Roles Tradicionales & Ruptura & Dependencia & Crítica & \\
\hline Dependencia & Sufrimiento & & Ruptura & Tradiciones \\
\hline Educación Pública & $\begin{array}{l}\text { Educación } \\
\text { Pública }\end{array}$ & & Sufrimiento & \\
\hline Trabajo & Trabajo & & $\begin{array}{l}\text { Educaci } \\
\text { Inter } \\
\text { Solvencia }\end{array}$ & $\begin{array}{l}\text { Vacional } \\
\text { ional } \\
\text { conómica }\end{array}$ \\
\hline
\end{tabular}

Además, el noviazgo es concebido como una relación que se toma en "serio", en la que hay confianza, apoyo, comprensión, sinceridad, fidelidad, afinidad y unión. El amor es el sentimiento más frecuentemente referido en los estratos bajo y medio. Aunque los atributos físicos tienen menor importancia para los adolescentes del estrato socio económico bajo y medio, ambos desean que su pareja sea atractiva. Tanto en el estrato bajo como en el medio la actividad sexual por una parte, es "inaceptable" en la adolescencia debido al embarazo o al sentimiento pecaminoso y por otra parte, es aceptable como relación afectuosa, estable y duradera, planeada con anticonceptivos y responsabilidad. Las mujeres de estratos bajo y medio temen, en el noviazgo, 
la pérdida de su independencia. Por ello, las pertenecientes al estrato bajo evitan el noviazgo en la institución, donde llevan una vida social más activa. Para las chicas los rumores dentro de la institución, llegan a constituirse en fuentes de sufrimiento y en ocasiones, de ruptura de sus amores.

Para los hombres pertenecientes a los estratos bajo y medio, el noviazgo es una oportunidad de reafirmación de masculinidad y ser reconocidos públicamente como heterosexuales, no solo ante sus pares, sino también ante sus padres.

\section{DISCUSIÓN}

Representaciones sociales convergentes en los tres estratos

El noviazgo hace parte de un conjunto de representaciones más amplio, donde el 'Proyecto de vida' es la categoría presente en hombres y mujeres de los tres estratos socioeconómicos. Es así, como aspiran alcanzar un nivel de formación profesional y la institución escolar cobra un valor importante como paso necesario hacia la educación superior. En todos los estratos, la formación profesional es central, ya sea para mantener o subir de nivel o para acceder a la autonomía e independencia familiar. También, el noviazgo aparece como una experiencia significativa, que favorecería el inicio de la actividad sexual. La diferencia de género es evidente. Las mujeres, expresan el control social sobre su sexualidad, desde todos los ámbitos sociales por el riesgo de embarazo con implicaciones en la realización del proyecto de vida. Los hombres, perciben permisividad social ya que mediante el noviazgo reafirman la masculinidad. No obstante, tanto hombres como mujeres prefieren postergar la actividad sexual a la edad adulta ó tener sexo con precaución. La conformación de una familia, es vista en la perspectiva de la juventud, una vez alcanzados logros personales: educativos, laborales y económicos (Cuadro 1).

Asimismo se ha documentado la necesidad de cambiar la conceptualización de la adolescencia como una etapa problemática, ligada a riesgos; por otra, donde se reconozca las condiciones que promueven el desarrollo positivo de los adolescentes (19-23). La perspectiva centrada en los riesgos sobreestima los comportamientos negativos y dirige la atención de los programas de intervención y políticas hacia aspectos problemáticos del adolescente y no a favorecer condiciones que puedan fomentar sus deseos de alcanzar un futuro mejor. 
Para los adolescentes de los tres grupos, el noviazgo es una experiencia positiva que se considera que tiene un carácter formal. Es decir, es un vínculo sólido, donde se espera compromiso, exclusividad, la expresión de sentimientos mutuos de comprensión, sinceridad y apoyo. Estos hallazgos, van en el mismo sentido del estudio de Díaz (24) sobre el noviazgo en adolescentes. El sentimiento más referido dentro del noviazgo por los adolescentes es el amor. Por ello, lo que más valoran los adolescentes en un novio(a) es su capacidad de dar afecto y la sinceridad en sus sentimientos, por encima de la apariencia física.

Un elemento convergente para los adolescentes de todos los estratos socioeconómicos, es que la edad de 20-30 años es sinónimo de soltería. Aparece como un periodo de consolidación del proyecto individual, de independencia y disfrute. No obstante, esta es igualmente la época en que se empieza a vislumbrar la conformación de una pareja, con miras al establecimiento de una familia, siempre y cuando hayan logrado alcanzar la estabilidad laboral y llevar una vida independiente y solvente.

Representaciones sociales convergentes por género en los tres estratos

Por otro lado, el embarazo aparece como una experiencia que trunca su proyecto de vida, limitando sus posibilidades de progreso educativo y económico especialmente para las adolescente del estrato bajo. Estos hallazgos son consistentes con otros estudios que destacan que los adolescentes conocen las implicaciones del embarazo en sus planes futuro (25), lo que contrasta con la perspectiva adulta que estigmatiza a la adolescencia como una etapa de irreflexiva, que no considera las consecuencias de sus actos, desorientada y problemática (26).

Como un aspecto diferencial se debe destacar que las adolescentes del estrato alto introducen, el consumo de alcohol como factor de riesgo, que puede conducir a que se tengan relaciones sexuales de forma irresponsable. Para los adolescentes de este estrato, el alcohol hace parte de sus actividades sociales. Este aspecto contrasta con las adolescentes del estrato bajo, donde el consumo de alcohol y las actividades nocturnas, tienen una connotación negativa, porque se cuestiona su valor como mujer de buenas costumbres y reservada al hogar (27).

Entre las adolescentes de estrato bajo, ser reconocidas como personas hogareñas, juiciosas y dedicadas a su estudio, les confiere valor y se torna 
fundamental para ser tomadas en serio en las relaciones amorosas. Estos hallazgos son consistentes con lo encontrado en un estudio sobre juventud en Bogotá (28), donde se reconoce que el imaginario sobre la mujer buena sigue pesando en lo que significa ser una niña de la casa que cumple con las obligaciones asignadas por la familia, permanece bajo el control paterno y tiene en la vida de hogar su realización personal. En los estratos medio y alto del estudio, la participación de las adolescentes en las actividades domésticas es mucho menor. Esto evidencia que la dimensión económica, marca diferencias, para este caso, frente al papel que cobra lo doméstico en la identidad femenina de las adolescentes. Los adolescentes varones de los tres estratos, participan mínimamente en las actividades domésticas.

Por otra parte, mientras las adolescentes ven en el noviazgo una red de apoyo importante ante los problemas de la vida, no sucede lo mismo en los adolescentes varones. Esto podría ser entendido, a la luz de las diferencias de cómo los y las adolescentes asumen el noviazgo. Las mujeres buscan más en una relación como una vía para dar y recibir amor, mientras que para los chicos se constituye en una forma de afianzar su masculinidad (29).

En cuanto a la dependencia afectiva, ésta es vista por las adolescentes como un aspecto negativo dentro de la relación de noviazgo, porque limita las relaciones sociales y los espacios de desarrollo individual. Las mujeres ven en la independencia, una necesidad de autonomía y espacio para su individualidad.

La presente investigación converge con aquellos estudios que dan cuenta sobre la pertinencia científica en torno a la necesidad de examinar los contextos y las instituciones para comprender cómo se constituyen las identidades de adolescentes, qué influencias tienen mayor impacto en sus decisiones relacionadas con salud, educación, trabajo, familia, etc., y así diseñar políticas y acciones que contribuyan a mejorar las condiciones de bienestar para esta población, propuestas desde el reconocimiento de heterogeneidad de las realidades de los adolescentes (30-31).

Las representaciones sociales que circulan en torno a las tareas psicosociales que se esperan para los adolescentes de los tres estratos socioeconómicos presentan un alto grado de convergencia, respecto a la culminación de su educación media y entrada a la educación superior para mejorar o mantener sus condiciones de vida. La educación aparece como un valor y un propósito fundamental en los procesos de formación en el presente 
y en las perspectivas futuras de los adolescentes. El proyecto de realización individual es una prioridad y posterga los procesos de conformación de familia*

\section{REFERENCIAS}

1. República de Colombia [ECOE Ediciones Colección Las Leyes de Colombia]. Ley General de Educación 115 de 1994.

2.DANE.[ Internet]. Disponible en: http://www.dane.gov.co/files/investigaciones/poblacion/ proyepobla06 20/7Proyecciones_poblacion.pdf. Consultado Diciembre del 2010.

3. Flórez, C, Soto, V. Fecundidad Adolescente y Desigualdad en Colombia y la Región de América Latina y el Caribe. Reunión de Expertos sobre Población y Pobreza en América Latina y el Caribe, Santiago de Chile; 2006.

4. Gaviria A. Los que suben y los que bajan. Educación y Movilidad Social en Colombia. Bogotá: Ediciones Alfaomega y Fedesarrollo; 2002.

5. Profamilia. Salud sexual y reproductiva en Colombia. Encuesta Nacional de Demografía y Salud del 2000. 1 ed. Bogotá; 2000.

6. Flórez C, Vargas E, Henao J, González C, Kassen D. Fecundidad Adolescente en Colombia: Incidencia, Tendencias y Determinantes. Un Enfoque de Historia de Vida. Documentos CEDE. Bogotá: Universidad de los Andes; 2004. pp. 84-117.

7. Vargas E, Barrera F. El papel de las relaciones padres-hijos y de la competencia psicosocial en la actividad sexual de los adolescentes. Documentos CESO Centro de Estudios Socioculturales e Internacionales departamento de Psicología. Bogotá: Universidad de los Andes; 2004. pp. 5-24.

8. Vargas E, Barrera F. Relaciones familiares y cogniciones románticas en la adolescencia: el papel mediador de la autoeficacia romántica. Revista de Estudios Sociales. Bogotá: Universidad de los Andes. 2005; (21); 27-35.

9. Vargas E, Berrera F. Adolescencia, relaciones románticas y actividad sexual. Una revisión. Revista Colombiana de Psicología. Bogotá: Universidad Nacional de Colombia. 2002; (011): $115-134$.

10. Vega L. Relación entre el apego y las relaciones románticas en la juventud. Documentos CESO Centro de Estudios Socioculturales e Internacionales Departamento de Psicología; 2005. pp. 3-47.

11. Wartenberg L. Embarazo precoz y embarazo adolescente en Colombia. Centro de investigaciones sobre dinámica social; 1999. pp. 31-55.

12. Kimmel I. La adolescencia: una transición del desarrollo. Primera edición. Barcelona: Ariel; 1998.

13. Banchs MA. Aproximaciones procesuales y estructurales al estudio de las representaciones sociales. Textos sobre representaciones sociales; 2000; 9 (3): 1-15.

14. Moscovici $S$. The fenomenon of social representations. In: Farr $R$ (Ed.) Social representations. Cambrige, Ed. De la maison des sciences del home; 1984.

15. Jodelet D. La representación social. Fenómenos, concepto y teoría. En: Moscovici S (Ed.). Psicología social II. Barcelona: Paídos; 1986.

16. De Rosa A. Le réseau d'associations comme méthode d'étude dans la recherche sur les représentations sociales : structure, contenus et polarité du champ sémantique. Les cahiers internationaux de psychologie sociale 1995; 28:97-123.

17. González LF. Algunos presupuestos generales del desarrollo de la investigación cualitativa en psicología. En: Toledo M, Medina G ((Ed.). Investigación cualitativa en psicología Rumbos y Desafíos. México: Internacional Thompson editores; 2000.

18. Ley 1090 de 2006 [Internet]. Disponible en: http://www.colpsic.info/resources/Ley109006.pdf. Consultado Enero del 2011. 
19. Serrano J. Juventud. Estado del arte, Bogotá 1990 -2000. Primera edición. Departamento Administrativo de Acción Comunal del Distrito. Bogotá: Colección Estados del Arte; 2003.

20. Perinat A. Los adolescentes de la era global. Comienzos del siglo XXI. En: Corral A, Crespo I (Ed.). El adolescente en el siglo XXI. Un enfoque psicosocial. Madrid: Ed. UOC; 2003.

21. Palma I, Abarca H, Moreno $\mathrm{C}$. Estrategias de prevención en salud sexual y reproductiva en jóvenes en América Latina y el Caribe: hacia una nueva síntesis de enfoques. Working Papers Series CST/ LAC; 2002 (14). pp.1-79.

22. Enrenfeld LN. Embarazo en adolescentes: aproximaciones social, cultural y subjetiva desde las jóvenes. Aproximaciones a la Diversidad Juvenil .En: Medina G. Aproximaciones a la Diversidad Juvenil. México: Colegio de México; 2000.

23. Martínez L. Mirando al futuro: desafíos y oportunidades para el desarrollo de los adolescentes en Chile. PSYKHE 2007; 16 (1):.3-14.

24. Díaz J. Identidad, adolescencia y cultura. Jóvenes secundarios en un contexto regional. Revista Mexicana de Investigación Educativa. 2006; 11 (29): 431-457.

25. Moreno S. Conducta sexual, conocimientos sobre el embarazo y necesidades percibidas con relación a la educación sexual, en adolescentes escolarizados. Cuaderno Venezolano de Sociología. 2006; 15 (4): 787-803.

26. Donas $S$. Adolescencia y juventud. Viejos y nuevos desafíos en los albores del nuevo milenio. En: Castillo M, Carazo R (Ed.). Adolescencia y juventud en América Latina. Costa Rica: Ed. Libro Universitario Regional; 2001.

27. Margulis M. Juventud: una aproximación conceptual. En: Adolescencia y juventud en América Latina. Costa Rica: Ed. Libro Universitario Regional; 2001.

28. Serrano J. Juventud. Estado del arte, Bogotá 1990 -2000. Departamento Administrativo de Acción Comunal del Distrito. Bogotá: Colección Estados del Arte; 2003.

29. Díaz J. Identidad, adolescencia y cultura. Jóvenes secundarios en un contexto regional. Revista Mexicana de Investigación Educativa. 2006; 11(29): 431-457.

30. Crockett L, Crouter, A. Pathways through adolescence: Individual development in relation to social contexts. Manhattan, NJ: Erlbaum;1995.

31. Nurmi J. Development of orientation to the future during early adolescent: A four-year longitudinal study and two cross-sectional comparisons. International Journal of Psychology. 1989; 24: 195-214. 